\title{
¿HACIA O EN LA SEGUNDA TRANSICIÓN DEMOGRÁFICA? \\ LOS CAMBIOS POBLACIONALES DE CHILE DESDE UN ENFOQUE DE GÉNERO
}

\author{
Ximena Vera Véliz
}




\section{XIMENA VERA VÉLIZ}

Socióloga por la Universidad de Chile y Master in Gender and Social Policy, por The London School of Economics and Political Science. Se ha desempeñado en departamentos de estudios y consultorías referidas a temas de género, estadísticas y políticas públicas, tanto para organismos públicos como privados. 


\section{¿HACIA O EN LA SEGUNDA TRANSICIÓN DEMOGRÁFICA? LOS CAMBIOS POBLACIONALES DE CHILE EXPLICADOS DESDE UN ENFOQUE DE GÉNERO ${ }^{1}$}

\section{RESUMEN}

Chile ha experimentado grandes cambios en su estructura poblacional durante las últimas décadas, lo que hace presumir que el país está entrando en un proceso de segunda transición demográfica. Para conocer si Chile comienza a vivir la segunda transición demográfica se analizan los tres principales componentes del cambio poblacional -fecundidad, mortalidad y migraciones- desde un enfoque de género. A lo largo del artículo se analiza cómo los cambios poblacionales generan efectos en ambos sexos, sobre todo en las mujeres. Se sostiene que solo el desarrollo de estudios poblacionales con enfoque de género, y de estudios de género que incorporen el componente poblacional, podrán ayudar a comprender (y afrontar) de mejor manera los cambios demográficos que se expresan actualmente en nuestro país.

Palabras clave: cambios poblacionales, fecundidad, migraciones, mortalidad, género.

\section{INTRODUCCIÓN}

El cambio demográfico de un determinado territorio puede referirse al cambio en el tiempo que se produce en el crecimiento de la población de aquel, en la distribución de esta o a los cambios en los componentes mayores del crecimiento poblacional, especialmente en la fertilidad (el número de hijas(os) por mujer), en la mortalidad (los riesgos de morir a una edad particular) y en las migraciones (desplazamientos geográficos de la población) (Mason, 1995; Haupt and Kane, 2001).

Es complejo determinar la dimensión temporal de los cambios demográficos, pues en general, exceptuando situaciones de guerras o graves desastres naturales o políticos, aquellos ocurren gradualmente. Si se analiza la composición poblacional de Chile en

1. Agradezco a mis estimados colegas Nurjk Agloni Jorquera y Víctor Orellana Calderón, por los valiosos comentarios que hicieron a este trabajo.

2. El estudio de los cambios poblacionales en Chile se basa en los datos generados por el Instituto Nacional de Estadísticas (INE) en la modalidad de datos censales de población y del registro de Estadísticas Vitales. Estas últimas se basan en registros administrativos, triangulados por información tanto del Registro Civil como del Ministerio de Salud. Cabe destacar que si bien el país cuenta con datos que permiten conocer tanto los hechos demográficos ya acaecidos como aquellos por ocurrir -por medio de proyecciones- el nivel de desagregación de estos hechos demográficos no permite realizar un 
los últimos sesenta años, se evidencia que esta ha entrado a un franco proceso de envejecimiento, cambiando notoriamente su pirámide poblacional (véanse, al respecto, los gráficos 1, 2 y 3). La población total del país en 1950 ascendía a 6.081.931 personas, mientras que sesenta años después se estima que la población prácticamente se triplicó, alcanzando 17.094.270 personas. En ambos períodos la distribución por sexo se ha mantenido más menos estable, siendo las mujeres un 50,47\% del total de la población en 1950 y un 50,5\% en 2010. El índice de feminidad ${ }^{3}$ ha tenido un leve aumento: aumentó de 101,89 en 1950 a 102,03 en 2010 (CEPAL, 2009). Este índice implica que al año 2010 se estima que en Chile existían 102,02 mujeres por cada cien hombres. El mayor número de mujeres en el país se explica tanto por causas biológicas como sociales: si bien es cierto nacen más hombres, ellos viven en promedio menos años. Esto se explica, entre otras causas, tanto porque los hombres son más susceptibles a algunas enfermedades como por su sobreexposición a muertes externas, como se verá más adelante.

La composición etaria de la población es el cambio más evidente a nivel de población en Chile. En 1950, el 36,7\% de la población tenía menos de 15 años, mientras que en el año 2010 se estima que solo un 14,5\% de ella se encontraba en el mismo rango de edad. De manera inversa, en 1950 un 4,3\% de la población tenía sobre 65 años, mientras que en el año 2010 dicho porcentaje se eleva hasta un 9\%. Las poblaciones menores de 15 años y mayores de 64 años se consideran dependientes, pues en teoría son económicamente inactivas. Será, por tanto, el segmento comprendido entre los 15 y los 64 años el que deberá sostener, mediante su fuerza de trabajo, la economía. Contrariamente a lo que podría pensarse, la relación de dependencia ${ }^{4}$ ha disminuido desde 1950 a la fecha. Esta disminución se debe principalmente a la abrupta caída de la fecundidad, lo que provocó una disminución evidente de la población infantil. La relación de dependencia en 1950 era de 69,4; en 1980 descendió a 62,8 y en 2010 disminuyó aun más, alcanzando un 46,0 (CEPAL, 2009).

análisis exhaustivo considerando características específicas como etnicidad o nivel socioeconómico de las personas.

3. Índice de feminidad: relación entre el número de mujeres y hombres. Su cálculo es: Población de mujeres/población de hombres *100 (INE, 2004).

4. Relación de dependencia: es un indicador de dependencia económica potencial; mide la población en edades "teóricamente" inactivas en relación a la población en edades "teóricamente" activas. Su cálculo es: Población de 0 a 14 años + población de 65 años y más / población de 15 a 64 años * 100 (CEPAL, 2009). 
Gráfico 1

POBLACIÓN EN CHILE EN 1950

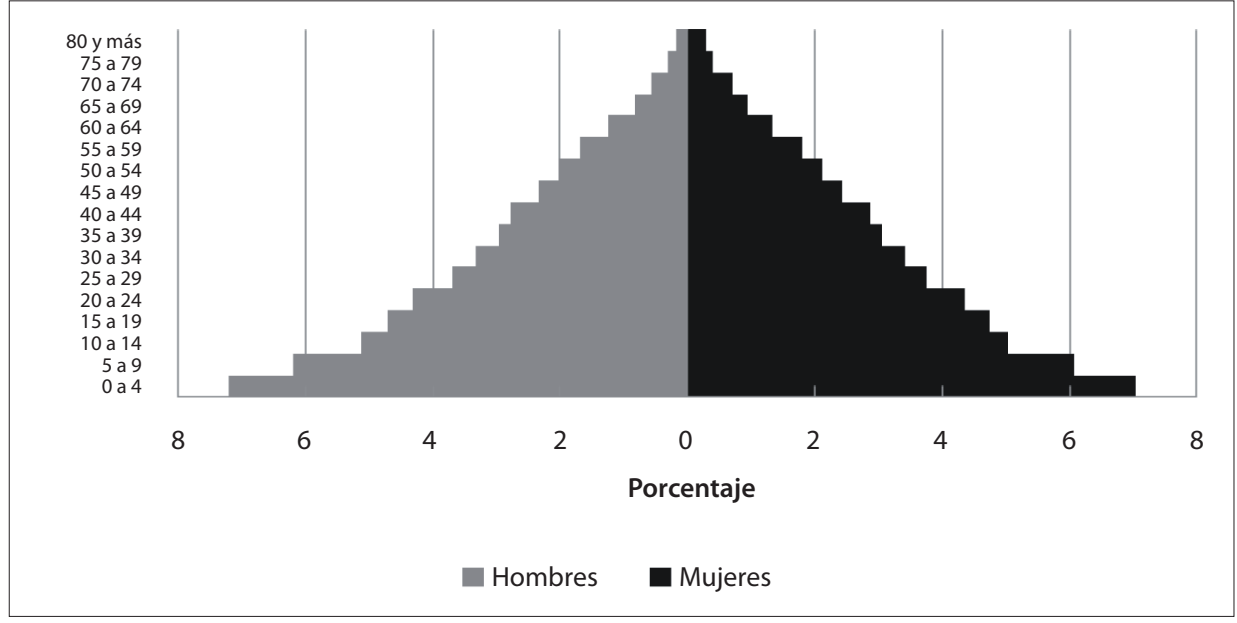

Fuente: Elaboración propia, según datos de INE y CEPAL (2004).

Gráfico 2

POBLACIÓN EN CHILE EN 1980

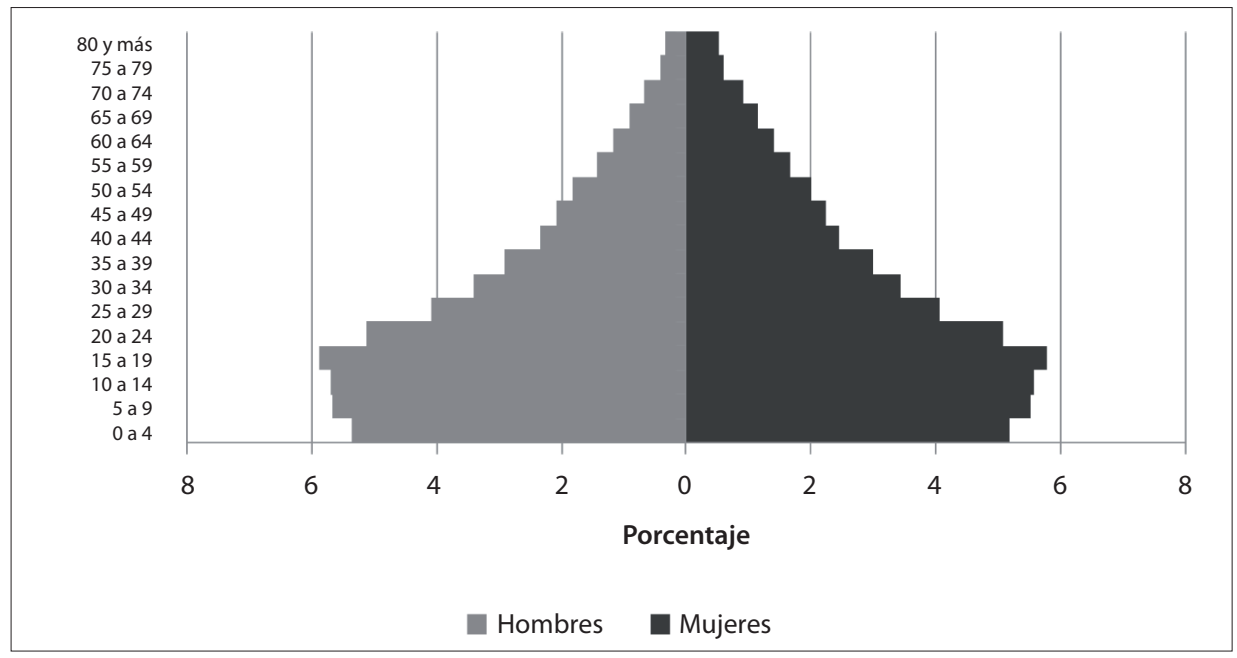

Fuente: Elaboración propia, según datos de INE y CEPAL (2004). 


\section{Gráfico 3}

POBLACIÓN EN CHILE EN 2010

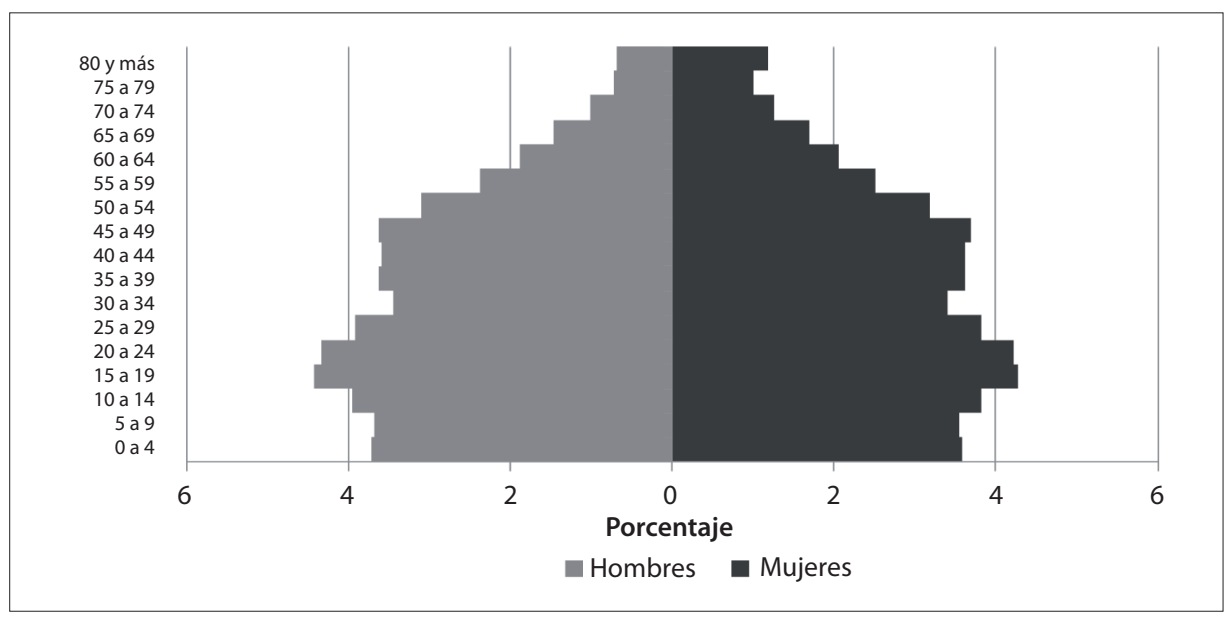

Fuente: Elaboración propia, según datos de INE y CEPAL (2004).

El cambio poblacional citado indica que Chile experimentó con rapidez la primera transición demográfica (Haupt and Kane, 2001): bajaron los niveles de fecundidad y de mortalidad, y aumentó la esperanza de vida. Según CEPAL (2010) esta transición se explica por los desarrollos médicos y sanitarios posteriores a la Segunda Guerra Mundial, los que en Chile se expresaron con mayor fuerza en la década del 60, debido a la implementación de políticas sociales y de salud. Estas provocaron una mejora sin precedentes en la calidad de vida de las personas, tanto por la disminución de la mortalidad como por el control de la natalidad. Dado que las evidencias estadísticas indican que la fecundidad y la mortalidad continuarán descendiendo en los años siguientes, expresando cambios poblacionales más menos acelerados, vale preguntarse si Chile se encuentra actualmente en el proceso de la segunda transición demográfica.

El concepto de segunda transición demográfica fue propuesto por los demógrafos Ron Lesthaeghe y Dirk van de Kaa, en el año 1986. Si bien en sus inicios el concepto generó reticencias en la academia ${ }^{5}, 20$ años más tarde ha sido ampliamente utilizado para explicar nuevas variaciones en la composición demográfica de algunos países (Billari y Liefbroer, 2004). Como componentes de la segunda transición demográfica se cuentan los siguientes factores: índices de fecundidad inferiores al nivel de reemplazo; aumento del celibato y de parejas que no desean tener descendencia; retraso de la primera unión;

5. Se le ha criticado en varios aspectos, sosteniendo que: no propone hipótesis claras; no es una "segunda" transición demográfica, sino más bien una transición secundaria; no es realmente demográfica, pues no tiene una formalización matemática rigurosa; y no puede ser descrita apropiadamente como una transición (Coleman, 2004). Es más, van de Kaa, uno de sus propios autores, sostiene que en vez de ser considerada transición debiese denominarse "revolución" (Billari y Liefbroer, 2004). 
postergación del (la) primer(a) hijo(a); aumento de las uniones consensuales como alternativa al matrimonio; aumento de los nacimientos y de la crianza fuera del matrimonio; mayor frecuencia de las rupturas matrimoniales (divorcio) y diversificación de las modalidades de estructuración familiar (Arriagada, 2004). Originalmente esta teoría no le asigna suficiente importancia a los cambios culturales que moldean esta transición. No obstante, algunas(os) autoras(es) se han encargado de complementarla, relevando los elementos sociológicos y normativos sobre las formas de constituir familia y sobre el comportamiento reproductivo (Mason, 1995).

Así, se establece que la segunda transición demográfica se vincula con profundos cambios valóricos, fuertemente relacionados con la noción de modernidad tardía propuesta por Giddens (1994 y 1995). Arriagada (2004) sostiene que los demógrafos que estudian este concepto y sus expresiones tienden también a relacionarlo con los "valores postmaterialistas" (Inglehart citado por van de Kaa, 2001), y más recientemente con postmodernización y postmodernidad (van de Kaa, 2001). La individualización o individuación es uno de los componentes típicos de esta era. Este concepto se refiere a que las personas intentan desligarse de modelos, normas y controles sociales, relevando la propia capacidad de acción y decisión (Beck y Beck-Gernsheim, 1998). Estas transformaciones valóricas se refieren tanto a formas de estar en el mundo como a la construcción de significaciones y proyecciones que los sujetos realizan tanto sobre su vida personal como sobre la situación colectiva del país.

Los cambios valóricos a los que se refiere el concepto de la segunda transición demográfica se insertan en sociedades que han vivido transformaciones que los propician $^{6}$. De todos modos no se puede generalizar sobre la linealidad que propone la teoría de la segunda transición demográfica. Si bien es cierto el desarrollo económico puede ser productor de los cambios demográficos ya citados, no es condición sine qua non. El propio van de Kaa (1997), citado en Medina y Fonseca (2003, pág. 33) sostuvo que el desarrollo de las teorías socioculturales sobre el descenso de la fecundidad obedece a la necesidad de explicar procesos de transición singulares, que no siguen los patrones de modernización de Occidente. Basta recordar que tanto Cuba como Chile presentan una tasa global de fecundidad inferior al nivel de reemplazo, pero ninguno de los dos países califica como país desarrollado. Los contextos de cada sociedad se encuentran llenos de factores que ayudan a explicar los cambios poblacionales.

Tanto los cambios demográficos como los cambios valóricos en los que estos se enmarcan afectan de forma diferenciada a mujeres y hombres. Es sabido, por ejemplo, que la esperanza de vida es mayor para las mujeres que para los hombres de su misma

6. En el caso de Chile las transformaciones en las últimas décadas son más menos evidentes, pese a que la construcción y la utilización de los indicadores siempre son sujetos de críticas. En términos globales, y solo por nombrar algunas de las transformaciones del país, vale recordar que, según datos del PNUD (2012), de 1980 a 2010 el PIB per cápita aumentó de US\$5.366 a US\$13.202 (US\$ de 2005); los años de educación promedio aumentaron de 6.4 a 9.7, respectivamente, y la población urbana aumentó del 81.2\% en 1980 a 89.1\% en 2010. Según MIDEPLAN (el antiguo Ministerio de Planificación y Cooperación, actualmente denominado Ministerio de Desarrollo Social) (2009) en el período 1990-2009 la pobreza se redujo desde el 38,6\% al 15,1\% de la población. 
cohorte. De la misma manera, debido a factores culturales de género, en Chile y en gran parte del mundo las mujeres son las principales "responsables" de la fecundidad y del subsecuente proceso de crianza, por lo que la decisión de tener o no hijas(os) tendrá una repercusión mayor en la vida de ellas que en la de los hombres. La utilización de la perspectiva de género en los asuntos de población hace más completa la propuesta teórica tras el concepto de segunda transición demográfica (Bernhardt, 2004), pues permite conocer cómo estos cambios afectan la vida de mujeres y hombres, identificando a su vez posibles inequidades demográficas. Estas inequidades pueden trabar el ejercicio de los derechos y la toma de decisiones individuales de las mujeres, a la vez que pueden actuar como resistencias en el proceso de transición demográfica, generando así nuevas inequidades (Heikel, 1996; Ariza y De Oliveira, 2000; Bernhardt, 2004). Es probable que los cambios demográficos afecten el sistema de género ${ }^{7}$ que impera en una sociedad, de la misma manera que cambios en este sistema afecten la composición de la población (Mason, 1995). No obstante lo anterior, conocer eventuales cambios en el sistema de género debido a los cambios poblacionales excede los objetivos de este trabajo.

En función de lo anterior, en el presente ensayo se analizará desde un enfoque de género los cambios poblacionales acontecidos en Chile en los últimos 60 años, donde todo parece indicar que el país se está adentrando en un proceso de segunda transición demográfica. Para ello se considerarán en el análisis los tres principales componentes del cambio demográfico: fecundidad, mortalidad y migraciones. El foco de análisis se pondrá principalmente en la fecundidad, debido a su principal relevancia tanto en la segunda transición demográfica como en especial en la vida de las mujeres. Se invertirá entonces el orden de presentación tradicional de los datos, comenzando de forma breve con migraciones y mortalidad, para finalizar analizando más en extenso la fecundidad.

\section{SOBRE LAS MIGRACIONES (Y LA FALTA DE DATOS POBLACIONALES EN EL PAÍS)}

Al estudiar cómo la migración ${ }^{8}$ ha incidido en la estructura poblacional del país emerge un grave obstáculo: los datos poblacionales sobre migración en Chile son realmente

7. Se entiende por sistema de género las expectativas socialmente construidas sobre el comportamiento de hombres y mujeres, que se encuentran en formas variables, en cada sociedad humana alguna vez conocida. Un sistema de expectativas de género prescribe una división del trabajo y de responsabilidades entre mujeres y hombres, y les establece diferentes derechos y obligaciones. Ya sea de forma intencional o como efecto colateral, crean a su vez desigualdades entre los sexos en el poder, su autonomía y bienestar. Usualmente, son las mujeres quienes se encuentran en desventaja. Aunque los sistemas de género cambian en el tiempo, muchas de sus expectativas constitutivas son fuertemente reforzadas por el Estado, la comunidad y a través de sanciones informales entre cualquier grupo social. Las reglas de comportamiento se les inculcan a los niños y niñas desde edades tempranas, y son a su vez las bases de la constitución de su personalidad (Rubin, 1975).

8. Por migración se entiende "el movimiento geográfico de personas a través de una frontera específica con fines de establecer una residencia permanente o semipermanente" (Haupt and Kane, 2001, pág. 32). 
escasos $^{9}$, y no permiten hacer un análisis considerando el enfoque de género. El INE (2004) sostiene que, según informes de proyecciones de población y de investigaciones sobre el tema, desde 1950 y hasta mediados de los años 70 los saldos migratorios netos $^{10}$ internacionales se muestran estables. La emigración fue más relevante que la inmigración, generando saldos netos migratorios que fluctuaron entre las -30.000 y las -40.000 personas.

Los datos disponibles indican que desde 1990 Chile revirtió el saldo neto migratorio internacional, convirtiéndose en país receptor de inmigrantes. Chile se vuelve receptor debido tanto a su estabilidad económica y política en comparación con el resto de los países de la región, como por el cierre de fronteras para la población migrante latinoamericana en Estados Unidos y en la Comunidad Europea (Stefoni, 2009). Así, se observa que en el período 1990-2000 el país recibió 200.000 personas, mientras que 50.000 decidieron emigrar de Chile. El saldo neto migratorio para el período fue de 150.000 personas, de las cuales se estima que 78.000 fueron mujeres y 72.000 fueron hombres (INE, 2004). Según datos del Departamento de Extranjería y Migración (2011), en los últimos diez años el aumento de permisos de visas ${ }^{11}$ ha sufrido un aumento explosivo: el año 2000 se solicitaron 18.325 visas, de las cuales 55,1\% correspondía a mujeres. Esta cifra se elevó en 2010 a 63.901 visas, donde 49,9\% de ellas fueron solicitadas, también, por mujeres.

\section{LA DISMINUCIÓN DE LA MORTALIDAD Y EL AUMENTO DE LA ESPERANZA DE VIDA}

La mortalidad presenta componentes de género relevantes de analizar. La Esperanza de vida al nacer ${ }^{12}$ es distinta para hombres y mujeres debido tanto a condicionantes biológicas como sociales. En todo el período 1950 a 2005 en Chile la esperanza de vida al nacer ha sido mayor para las mujeres que para los hombres. En 1950 el promedio de años fue de 54,8; se esperaba que las mujeres viviesen en promedio hasta los 56,7 años y los hombres hasta los 52,9 años. Cincuenta años después la esperanza de vida al nacer representaba un promedio de 77,7 años; se esperaba que las mujeres viviesen hasta los 80,8 años en promedio, y que los hombres lo hiciesen hasta los 74,8 años. En los últi-

9. No existen datos poblacionales referidos a migración para el período intercensal 2002-2012, lo que vuelve especialmente complejo el análisis de este componente, especialmente considerando que en los últimos años Chile se ha convertido en un foco de atracción de inmigrantes de países vecinos.

10. Saldo migratorio neto: "Representa el componente migratorio del crecimiento total de una población. La magnitud de este saldo se mide calculando la diferencia media anual entre inmigrantes y emigrantes de una población" (CEPAL, 2010, pág. 254).

11. Se excluyen las visas de turismo.

12. Esperanza de vida al nacer: "Representa la duración media de la vida de los individuos que integran una cohorte hipotética de nacimientos, sometidos en todas las edades a los riesgos de mortalidad del período en estudio" (CEPAL, 2010, pág. 247). 
mos cincuenta años, se espera que las mujeres vivan en promedio seis años más que los hombres de su misma cohorte. La siguiente tabla ilustra lo anterior.

Tabla 1

ESPERANZA DE VIDA AL NACER (EN AÑOS), POR SEXO. PERÍODO 1950 A 2005

\begin{tabular}{|l|c|c|c|c|c|c|}
\hline & $1950-1955$ & $1960-1965$ & $1970-1975$ & $1980-1985$ & $1990-1995$ & $2000-2005$ \\
\hline Mujeres & 56,77 & 60,95 & 66,8 & 75,89 & 77,35 & 80,80 \\
\hline Hombres & 52,91 & 55,27 & 60,46 & 69,59 & 71,45 & 74,80 \\
\hline Total & 54,80 & 58,05 & 63,57 & 72,68 & 74,34 & 77,74 \\
\hline
\end{tabular}

Fuente: INE y CEPAL, 2004

El aumento de la esperanza de vida de la población trae consecuencias para el conjunto de la sociedad, pero específicamente produce la acentuación de las inequidades de género que imperan en la sociedad chilena. Solo por nombrar dos: las mujeres viven hasta edades más avanzadas, lo que afecta las pensiones que puedan conseguir, empobreciéndolas de manera radical (debido a su menor participación laboral tienen menos cotizaciones previsionales; también las mujeres imponen en el sistema previsional por menos tiempo y sus sueldos son considerablemente más bajos que los de sus pares varones, por lo que sus cotizaciones también son menores. Como viven más, sus pensiones son inferiores que las de los hombres). Por otra parte, los cuidados de la cada vez mayor población de tercera edad que requiere de cuidados, recaen en el trabajo no remunerado de las mujeres. Como se verá más adelante, la obligación de tener que cuidar a parientes ancianos(as) propios o de la pareja limita el acceso de las mujeres a otros ámbitos de la vida social, incluyendo su disponibilidad de tiempo libre.

Sumado al aumento de la esperanza de vida, la mortalidad infantil descendió de 120 a 8 muertes por cada mil nacimientos desde el período 1950-1955 al quinquenio 2000-2005, respectivamente. CEPAL (2010) sostiene que la tendencia a la disminución de la mortalidad se explica por los avances de la medicina, el desarrollo de sistemas de atención de la salud más apropiados, las mejoras de las condiciones de vida de la población y el cambio de los estilos de vida (pese a que no todos estos factores conducen al descenso de la mortalidad). En Chile el descenso de la mortalidad se enmarca dentro de una "transición epidemiológica": ha disminuido la mortalidad producto de evitar las defunciones causadas por enfermedades transmisibles. Ello produce que cambie el perfil de las defunciones por causas y grupos de edad y se incremente el peso relativo de las muertes por enfermedades crónicas y degenerativas, más frecuentes en los adultos.

Las principales causas de muerte en Chile presentan diferencias y a la vez similitudes entre mujeres y hombres. En el período 1990 a 2007 las dos principales causas de muerte para ambos sexos fueron aquellas relativas a las enfermedades del sistema circulatorio, seguidas por tumores malignos. La tercera principal causa de muerte es distinta para mujeres y hombres: para ellas son las enfermedades respiratorias (causa que ocupa el cuarto lugar en el caso de los hombres), mientras que en el caso de ellos 
el tercer principal motivo de fallecimiento son las muertes debido a causas externas ${ }^{13}$. La tasa bruta de mortalidad ${ }^{14}$ de los hombres según esta causa en el período 20052007 fue de 76,2 hombres por cada cien mil habitantes. En cambio, esta causa es el sexto gran motivo de fallecimiento de las mujeres. Durante el período señalado la tasa bruta de mortalidad de las mujeres según esta causa fue de 19,8 mujeres por cada cien mil habitantes.

Tabla 2

TASAS BRUTAS DE MORTALIDAD, POR SEXO, DE LAS PRINCIPALES CAUSAS DE MUERTE EN EL PERÍODO 1990-1992 A 2005-2007 EN CHILE

\begin{tabular}{|l|c|c|c|c|}
\hline \multirow{2}{*}{ Causas de muerte } & \multicolumn{2}{|c|}{ Mujeres } & \multicolumn{2}{c|}{ Hombres } \\
\cline { 2 - 5 } & $1990-1992$ & $2005-2007$ & $1990-1992$ & $2005-2007$ \\
\hline Enf. Sistema circulatorio & 158,84 & 148,04 & 161,24 & 156,31 \\
\hline Tumores malignos & 107,66 & 123,12 & 109,11 & 133,54 \\
\hline Enf. Sistema respiratorio & 58,11 & 50,59 & 71,23 & 52,17 \\
\hline Causas externas & 28,92 & 19,79 & 109,59 & 76,23 \\
\hline
\end{tabular}

Fuente: INE, 2009.

Las muertes según causas externas se explican principalmente por factores sociales y culturales: los hombres son socializados incentivándoles conductas que los vuelven susceptibles de sufrir consecuencias graves para su salud. Se les inculca que deben ser independientes, agresivos, actuar de forma temeraria y a veces violenta en diversos ámbitos de su vida (Conell, 1995). Así, su exposición a accidentes de tránsito, peleas y adicciones se incrementa de forma exponencial en comparación con las mujeres de su misma cohorte. De la misma manera, debido a la división sexual del trabajo las faenas que pueden ser de alto riesgo para la vida (trabajos como mineros, obreros operarios de maquinaria pesada, bomberos, pescadores, entre otros) son realizadas principalmente por hombres. Si se analizan los datos relativos a causas de muertes externas según sexo, se aprecia que en el año 2009 un 78,7\% de este tipo de muertes ocurrió a hombres. El peso porcentual de las muertes masculinas debido a estas causas se incrementa en el grupo etario de entre 30 y 44 años, donde un $84,5 \%$ del total de estas muertes en este grupo etario correspondió a hombres. La Tabla 3 ilustra lo anterior.

13. Comprende accidentes de todo tipo, envenenamiento, agresiones, entre otras.

14. Tasa bruta de mortalidad: "Es el cociente entre el promedio anual de defunciones ocurridas durante un período determinado y la población media de ese período" (CEPAL, 2010, pág. 247). Dicho en otras palabras, es el número de muertes por cada 1.000 habitantes durante un año determinado. En Chile, el INE presenta esta tasa a razón de 100.000 habitantes. Así, una tasa bruta de mortalidad de 76,2 indica que mueren 76,2 personas por cada 100.000 habitantes. 


\section{Tabla 3}

NÚMERO DE MUERTES POR CAUSA EXTERNA Y PESO PORCENTUAL DE LOS HOMBRES SOBRE EL TOTAL, POR SEXO Y GRUPOS DE EDAD EN CHILE. AÑO 2009

\begin{tabular}{|l|c|c|c|c|c|}
\hline & $15-29$ años & $30-44$ años & $45-59$ años & $\begin{array}{c}60 \text { años y } \\
\text { más }\end{array}$ & Total \\
\hline Mujeres & 324 & 286 & 292 & 759 & 1.661 \\
\hline Hombres & 1.592 & 1.560 & 1.507 & 1.495 & 6.154 \\
\hline Total & 1.916 & 1.846 & 1.799 & 2.254 & 7.815 \\
\hline \% de hombres sobre el total & 83,1 & 84,5 & 83,8 & 66,3 & 78,7 \\
\hline
\end{tabular}

Fuente: INE, 2010.

Como se observó, los indicadores de mortalidad tienen especificidades por sexo. Estas deben ser conocidas para poder controlarlas y así evitar inequidades demográficas y sus consecuencias (tanto para evitar las muertes tempranas de los hombres como para mejorar las condiciones de vida de la población femenina envejecida).

\section{EL DESCENSO SOSTENIDO DE LA FECUNDIDAD EN CHILE Y LOS FACTORES DE GÉNERO QUE CONTRIBUYEN A EXPLICARLO}

Por fecundidad se entiende "la frecuencia de los nacimientos que ocurren en el seno de grupos o subgrupos humanos en edad de procrear" (CEPAL, 2008, pág. 184). En Chile, se considera que las mujeres en edad de procrear son todas aquellas que se ubican en el rango de 15 a 49 años (INE, 2006). Debido a que el número absoluto de nacimientos es poco indicativo, para analizar la fecundidad se considera la Tasa Global de Fecundidad (TGF) ${ }^{15}$. En los últimos 50 años esta tasa ha bajado abruptamente en el mundo ${ }^{16}$. La tabla siguiente indica que la TGF de Chile ha sido, en general, más baja que la tasa promedio del mundo y que la de América Latina. En 1955 América Latina (no incluye el Caribe) tenía una TGF de 5,89 hijas(os) por mujer, mientras que la de Chile era de 4,95 hijas(os) por mujer. Cincuenta años después la TGF se redujo aun más, siendo de 2,37 hijas(os) por mujer en América Latina, y en Chile se estima que se situó bajo

15. La Tasa Global de Fecundidad es el número de nacimientos vivos por cada grupo de 1.000 mujeres entre las edades de 15 a 49 años durante un año determinado. La TGF indica el número promedio de hijas(os) vivas(os) que una mujer hubiese tenido al final de sus años reproductivos (Haupt and Kane, 2001).

16. Por supuesto, al hablar del "mundo" se hace referencia a la Tasa Global de Fecundidad a nivel planetario, lo que sin duda enmascara las grandes diferencias por región. No obstante lo anterior, incluso en África, la región que históricamente ha tenido una TGF mayor, esta ha disminuido notoriamente: en 1955 la TGF de este continente era de 6,75 hijos(as) por mujer, disminuyendo a 4,67 en el quinquenio 2005-2010 (CEPAL, 2009). 
el nivel de reemplazo: 1,94 hijas(os) por mujer. En los últimos cincuenta años, la tasa global del país se redujo en un $60,8 \%$.

Tabla 4

TASA GENERAL DE FECUNDIDAD (TGF) EN AMÉRICA LATINA Y CHILE

\begin{tabular}{|l|c|c|c|c|c|c|c|c|c|c|c|c|}
\hline & 1955 & $\begin{array}{c}1955- \\
1960\end{array}$ & $\begin{array}{c}1960- \\
1965\end{array}$ & $\begin{array}{c}1965- \\
1970\end{array}$ & $\begin{array}{c}1970- \\
1975\end{array}$ & $\begin{array}{c}1975- \\
1980\end{array}$ & $\begin{array}{c}1980- \\
1985\end{array}$ & $\begin{array}{c}1985- \\
1990\end{array}$ & $\begin{array}{c}1990- \\
1995\end{array}$ & $\begin{array}{c}1995- \\
2000\end{array}$ & $\begin{array}{c}2000- \\
2005\end{array}$ & $\begin{array}{c}2005- \\
2010\end{array}$ \\
\hline Mundo & 5,02 & 4,96 & 4,98 & 4,90 & 4,47 & 3,92 & 3,58 & 3,38 & 3,05 & 2,80 & 2,65 & 2,55 \\
\hline AL & 5,89 & 5,94 & 5,98 & 5,57 & 5,05 & 4,50 & 3,94 & 3,42 & 3,03 & 2,74 & 2,53 & 2,37 \\
\hline Chile & 4,95 & 5,49 & 5,44 & 4,44 & 3,63 & 2,80 & 2,67 & 2,65 & 2,55 & 2,21 & 2,0 & 1,94 \\
\hline
\end{tabular}

Fuente: INE y CEPAL, 2004; INE, 2006.

El hecho que en el quinquenio 2005-2010 la TGF haya sido menor a 2, la ubica bajo el nivel de reemplazo. Es decir, bajo el nivel de fecundidad donde las mujeres dentro de la misma cohorte tienen suficientes hijas (os) (en promedio) para "reemplazarse" a sí misma y a una pareja, dentro de la población (Haupt and Kane, 2001). La disminución de la TGF a niveles inferiores al nivel de reemplazo es una tendencia que se expresa en algunos países desarrollados. Si bien en América Latina no existen países desarrollados, dos países han alcanzado en el quinquenio 2005-2010 un valor por debajo del nivel de reemplazo: Cuba, que lo alcanzó hace 30 años, y Chile, quien lo hizo recientemente (CEPAL, 2008).

La TGF toma valores distintos en cada país, y dentro de cada uno toma también valores diferentes según las características sociodemográficas de las madres. Hasta ahora se han mencionado las tasas globales promedio de fecundidad, pero es evidente que al analizar la TGB según características como el origen étnico o el nivel socioeconómico de las madres se encontrarán valores diferenciados en los distintos grupos analizados. Lamentablemente no existen datos desagregados que permitan analizar la TGF de forma histórica según estas características. A este respecto solo se sabe que en el año 2002 la TGB de las mujeres indígenas (2,1 hijas(os) por mujer) se estimaba levemente superior al de las mujeres no indígenas del país (2,0 hijas(os) por mujer) (INE, 2002). Las tasas de fecundidad según nivel socioeconómico (Larrañaga, 2006) indican que al año 2000 la TGB del grupo etario de mujeres entre 35 y 39 años del primer cuartil (más bajo) era de 2,88 hijas(os) por mujer, mientras que el mismo grupo de sexo y edad perteneciente al más alto nivel socioeconómico presentaba una tasa de 1,91 hijas(os) por mujer.

Si se analiza la TGF de Chile según los grupos etarios de las madres (véase Tabla 5), históricamente el grupo etario de mujeres entre 25 y 29 años es el que presenta la más alta TGF, seguido por el grupo de entre 20-24 años. Es decir, la reproducción se presenta principalmente entre los 20 y los 30 años de las mujeres. 


\section{Tabla 5}

TASA GENERAL DE FECUNDIDAD EN CHILE Y SU DISMINUCIÓN PORCENTUAL SEGÚN GRUPOS ETARIOS DE MUJERES. PERÍODO 1950-1955 A 2000-2005

\begin{tabular}{|l|c|c|c|}
\hline & $1950-1955$ & $2000-2005$ & Disminución de la TGF \\
\hline $15-19$ años & 0,08 & 0,0616 & $23 \%$ \\
\hline $20-24$ años & 0,215 & 0,0932 & $56,7 \%$ \\
\hline 25 a 29 años & 0,245 & 0,1004 & $59,0 \%$ \\
\hline 30 a 34 años & 0,21 & 0,0829 & $60,5 \%$ \\
\hline 40 - 34 años & 0,145 & 0,0478 & $67,0 \%$ \\
\hline 45 a 49 años & 0,075 & 0,0133 & $82,3 \%$ \\
\hline TGF & 0,02 & 0,0008 & $96,0 \%$ \\
\hline
\end{tabular}

Fuente: INE y CEPAL, 2004.

Con el transcurso de los años la TGF ha disminuido en todos los grupos, pero en proporciones distintas. En los grupos de edad sobre los 30 años la disminución ha sido superior al 60\%, mientras que en el segmento adolescente (15 a 29 años) disminuyó apenas un $23 \%$. Esto último implica que la típica creencia sobre el aumento de la maternidad adolescente es solo en parte verdad: la tasa global de fecundidad para este grupo etario disminuyó, pero a un nivel muy bajo en comparación a la disminución de la tasa del resto de los grupos. Ello provoca que al considerar la participación porcentual del grupo adolescente sobre el total de nacimientos, la fecundidad adolescente aumente. La Tabla 6 indica claramente que la participación porcentual de la maternidad adolescente sobre el total de maternidades pasa de un 8,08\% en el quinquenio 1950-1955 a un 14,81\% en el quinquenio 2005-2010. De manera inversa, la maternidad en grupos etarios de mujeres de 35 años y más ha caído de forma sostenida desde 1950 hasta el último quinquenio analizado: bajó desde un 24,25\% a un 14,48\%.

Tabla 6

PORCENTAJE DE NACIMIENTOS SEGÚN EDAD DE LA MADRE. PERÍODO 1980-1985 A 2005-2010

\begin{tabular}{|l|c|c|c|c|c|c|c|c|c|c|c|}
\hline & $\begin{array}{c}1950- \\
1955\end{array}$ & $\begin{array}{c}1955- \\
1960\end{array}$ & $\begin{array}{c}1960- \\
1965\end{array}$ & $\begin{array}{c}1965- \\
1970\end{array}$ & $\begin{array}{c}1970- \\
1975\end{array}$ & $\begin{array}{c}1975- \\
1980\end{array}$ & $\begin{array}{c}1980- \\
1985\end{array}$ & $\begin{array}{c}1985- \\
1990\end{array}$ & $\begin{array}{c}1990- \\
1995\end{array}$ & $\begin{array}{c}1995- \\
2000\end{array}$ & $\begin{array}{c}2000- \\
2005\end{array}$ \\
\hline $\begin{array}{l}15-19 \\
\text { años }\end{array}$ & 8,08 & 7,99 & 8,08 & 9,32 & 11,58 & 12,7 & 12,79 & 12,1 & 12,08 & 13,53 & 14,81 \\
\hline $\begin{array}{l}20-34 \\
\text { años }\end{array}$ & 67,67 & 69,01 & 69,4 & 69,19 & 70,93 & 72,02 & 71,84 & 73,91 & 74,9 & 72,18 & 70,72 \\
\hline $\begin{array}{l}35 \text { años } \\
\text { y más }\end{array}$ & 24,25 & 23 & 22,52 & 21,49 & 17,49 & 15,28 & 15,39 & 13,98 & 13,02 & 14,29 & 14,48 \\
\hline
\end{tabular}

Fuente: INE y CEPAL, 2004. 
Contrariamente a lo que postula la segunda transición demográfica, la edad promedio de la madre al momento de tener la primera hija o el primer hijo no ha variado significativamente en los últimos años en Chile, manteniéndose cercana a los 23,3 años en promedio (INE, 2003).

En la decisión de las mujeres sobre cuándo permitir la ocurrencia de un embarazo en su propio cuerpo intervienen diversos factores: la pareja, la posibilidad de desarrollo profesional y la biología (el calendario biológico) entre otros (económicos, influencia social, etc.) (Paredes, 2008). El número de hijas(os) que una pareja tendrá puede ser producto tanto de decisiones racionales de acuerdo (relativamente) mutuo entre las partes, decisiones solo de la mujer o simplemente la expresión azarosa del ejercicio de la sexualidad. Sin embargo, al considerar posibles explicaciones sobre el descenso de la fecundidad se debe tener siempre en cuenta que finalmente sobre las mujeres recae la decisión final de tener una hija o hijo, por medio de un (relativo) equilibrio (o justificación o aceptación) de los factores intervinientes.

Las explicaciones sobre el descenso de la fecundidad pueden ser múltiples: pueden estar basadas en decisiones individuales de carácter económico (costo/beneficio) o de carácter, por denominarlo de alguna manera, social. En el primero de los casos primarían las decisiones de tipo monetario: aumentar el número de hijas(os) implica aumentar el gasto y, en teoría, una disminución de la calidad de vida que se le podría brindar a las hijas(os) si es que su número fuese mayor. Las condicionantes sociales se relacionan con los valores culturales dominantes socialmente. La primacía ética y moral de valores más bien individualistas como la realización individual, la libertad personal y la emancipación, influirían en las (nuevas) formas de conformar familia y en la regulación de la fecundidad. Laesthague et al. (1980; 1992), citados en Medina y Fonseca (2003, pág. 33), plantean que estos cambios en los valores no son necesariamente producto de la modernización económica, sino que pueden existir debido a cambios meramente sociales y culturales. Este punto es de especial relevancia al analizar el contexto chileno, pues si bien el país ha mejorado varios de sus indicadores macroeconómicos en los últimos años, la desigualdad social es permanente. No obstante, los segmentos medios y altos de Chile han estado en frecuente contacto con normas y valores preponderantes en otras regiones del mundo, por lo que existen crecientes aspiraciones de consumo, a la vez que el papel de las mujeres en la sociedad ha ido cambiando paulatinamente (Garvia y Rojas, 2001; Arriagada, 2004).

Dentro de los cambios sociales y culturales se cuentan las mejoras en la situación de las mujeres (mayor escolaridad, acceso a métodos anticonceptivos, por ejemplo). También es probable, como sostiene Bernhardt (1993), citada en Paredes (2008), que la "emancipación" de las mujeres haya posibilitado la disminución en el número de hijas(os) que se tienen. Esta autora sugiere que el descenso de la fecundidad se explica debido al conflicto entre crianza de las hijas e hijos y el trabajo remunerado de las mujeres. Mientras en Occidente ellas se han incorporado cada vez en mayor medida al trabajo remunerado, los hombres no han aumentado en la misma medida su participación en la crianza de la prole, por lo que en términos de costo/beneficio el tener (más) hijas(os) les impediría a las mujeres poder participar de mejor manera tanto en el 
mundo del trabajo remunerado (principalmente) como en otras áreas de la vida social (participación social, política, uso del tiempo libre, por citar solo algunos). En definitiva, actualmente las mujeres buscan la realización personal no solo en el ámbito privado, sino también en el público.

Aplicada en el caso chileno, esta teoría en parte puede explicar el descenso de la fecundidad. Si bien la tasa de participación laboral de las mujeres chilenas se encuentra dentro de las más bajas de América Latina (42\% el año 2009, según la encuesta CASEN), las mujeres económicamente activas del país tienen menos hijas(os) en promedio que aquellas económicamente no activas ${ }^{17}$. Los datos censales indican que tanto en 1982 como en 1992 las mujeres activas tenían un promedio de 1,6 hijos(as), mientras que en 2002 el promedio disminuyó a 1,5. Las mujeres inactivas, por su parte, en 1982 tenían en promedio 3,3 hijos(as), mientras que en 2002 el promedio descendió a 2,5 hijas(os) en promedio (INE, 2006).

Si se analiza el tiempo que las mujeres destinan a las labores de crianza y reproducción del hogar, se hace evidente que las tareas domésticas y de cuidado limitan la posibilidad de las mujeres de acceder al mundo público (trabajo remunerado y participación política) y de ejercer sus derechos (Lister, 1997). En Chile el desarrollo del trabajo no remunerado ${ }^{18}$ es realizado principalmente por las mujeres, independientemente de si participan o no en el mercado laboral de forma activa. Los datos de la Encuesta de Uso del Tiempo, realizada en 2007 por el INE, grafican lo anterior:

\section{Tabla 7}

TASA DE PARTICIPACIÓN Y PROMEDIO DE HORAS DIARIAS DEDICADAS A TRABAJO DOMÉSTICO NO REMUNERADO*, POR SEXO Y SEGÚN CONDICIÓN DE ACTIVIDAD ECONÓMICA. SOLO GRAN SANTIAGO

\begin{tabular}{|l|c|c|c|c|c|c|}
\hline \multirow{2}{*}{$\begin{array}{l}\text { Condición de acti- } \\
\text { vidad económica }\end{array}$} & \multicolumn{2}{|c|}{ Mujeres } & \multicolumn{2}{c|}{ Hombres } & \multicolumn{2}{|c|}{ Total } \\
\cline { 2 - 7 } & \% Part. & $\begin{array}{c}\text { Prom. Hrs. } \\
\text { diarias TDNR }\end{array}$ & \% Part. & $\begin{array}{c}\text { Prom. Hrs. } \\
\text { diarias TDNR }\end{array}$ & \% Part. & $\begin{array}{c}\text { Prom. Hrs. } \\
\text { diarias TDNR }\end{array}$ \\
\hline Ocupados/as** & 75 & 4 & 41.2 & 2.8 & 54.5 & 3.4 \\
\hline No Ocupados/as*** & 87.5 & 5.4 & 51.8 & 2.9 & 76.1 & 4.9 \\
\hline Total & 82.1 & 4.8 & 44.2 & 2.8 & 63.8 & 4.2 \\
\hline
\end{tabular}

Fuente: Elaboración propia. INE, Encuesta de Uso del Tiempo, 2007

* Incluye tareas domésticas en el hogar y labores de cuidado.

** Ocupados/as: Personas que deben "haber trabajado al menos una hora en alguna actividad económica durante la semana de referencia y haber devengado un ingreso por ese trabajo (recibió o recibirá un pago por el servicio prestado, en efectivo y/o en especie)" (INE, 2009).

*** No ocupados/as: Categoría creada para agrupar a las personas en edad de trabajar que no participan en el trabajo remunerado. Está compuesta por la suma de las Personas Desempleadas + Personas Buscando Trabajo por Primera Vez + Personas No Económicamente Activas (pero en edad de trabajar), considerando que cada uno de estos grupos no participan en el trabajo remunerado.

17. Sin duda en estos casos intervienen variables como nivel socioeconómico y nivel educacional, entre otros.

18. El trabajo doméstico no remunerado comprende tareas domésticas (asear, lavar, planchar, cocinar, etc.) y de cuidado (de niños(as), personas de la tercera edad o personas con discapacidades). 
Como lo indica la tabla precedente, tan solo un 41,2\% de los hombres que están ocupados participan en Trabajo Doméstico No Remunerado (TDNR), destinando a este tipo de trabajo un promedio de 2,8 horas diarias. Por contraste, un $75 \%$ de las mujeres ocupadas participa en TDNR, destinando un promedio de 4 horas diarias a él. En el caso de las personas no ocupadas la participación en Trabajo Doméstico No Remunerado aumenta, pero el aumento prácticamente no permite acortar la brecha de participación entre hombres y mujeres: un 51,8\% de los hombres No Ocupados realiza TDNR, mientras un $87,5 \%$ de las mujeres de la misma categoría lo realiza. En cuanto al promedio de horas diarias destinadas a TDNR, la brecha entre hombres y mujeres no ocupados(as) es aun mayor: 2,9 horas en el caso de los hombres y 5,4 horas diarias en promedio son destinadas por las mujeres a TDNR.

De esta manera, cobra fuerza la propuesta teórica de Bernhardt (1993, citada en Paredes (2008)) sobre utilizar el paradigma de género para explicar los cambios en la fecundidad. Es probable que si en Chile primase un modelo de equidad de género en el que hombres y mujeres adoptasen roles laborales y domésticos similares, aunque los patrones culturales fuesen de carácter individualista, la fecundidad no hubiese caído a los niveles actuales. Uno de los factores que ayudarían a que la Tasa Global de Fecundidad vuelva a ser superior al nivel de reemplazo, sería un cambio cultural que aborde diversos ámbitos: desde que los hombres se hagan parte de manera equitativa en las labores reproductivas (de crianza), hasta que dicho cambio cultural abarque el comportamiento y la orientación de las instituciones (Mac Donald (2000), citada en Bernhardt, 2004). Las instituciones debiesen basarse en un sistema en que ambas partes, madres y padres, contribuyan de forma equitativa en la crianza, algo que no realizan actualmente. Tal como sostiene Paredes (2008, pág. 5):

Existe un conflicto entre las necesidades producidas por el proceso de individualización y las estructuras institucionales que, en muchos aspectos, mantienen su carácter estamental en las sociedades modernas, perpetuando la desigualdad entre hombres y mujeres. Los problemas que la sociedad de mercado impone no se pueden resolver mediante el mantenimiento de las formas de vida y las estructuras institucionales de una sociedad de mercado a medias.

Lo anterior se refuerza al citar un ejemplo: el año 2011 fue promulgada en el país la Ley de Postnatal ${ }^{19}$. Esta ley aumenta el período de post natal de dos a seis meses, pero solo para las mujeres ${ }^{20}$, dejando al padre única y exclusivamente con el período previo de 5 días libres desde el nacimiento de su hija o hijo. Si bien es cierto se incluye la opción de ceder parte del post natal al padre, el hecho de ser una opción limita su

19. Ley No20.545 de la República de Chile.

20. Y también solo para algunas mujeres: ellas deben haber tenido un último contrato que haya sido a plazo fijo por obra o faena, deben tener un año de filiación y al menos ocho cotizaciones continuas o discontinuas en los últimos 24 meses. 
materialización ${ }^{21}$. Es claro que el período de puerperio afecta directamente a las mujeres debido a condicionantes biológicas, no obstante la extensión del post natal a seis meses solo para mujeres implica que el Estado reconoce la primera crianza netamente como una labor femenina, situación que impide la participación de las mujeres en igualdad de condiciones con los hombres en los diversos ámbitos de la vida social. Si bien el permiso de post natal actual facilitaría la vida de las (pocas) mujeres que pueden optar a él, resulta un tanto iluso creer que la mejora en este permiso contribuirá a aumentar de manera significativa las tasas de fecundidad, tal como lo sostuvo el presidente de la República al referirse a aquel: "Yo espero que este nuevo postnatal, esta nueva forma de compatibilizar el trabajo y la familia, permita un repunte de la natalidad, de la vida, de la maternidad en nuestro país" (El Mostrador, 2012).

Por otra parte, existen otros hechos demográficos que eventualmente podrían influir en el descenso de la natalidad ${ }^{22}$. Sin duda estos hechos demográficos dan cuenta de los cambios sociales y culturales vividos en el país, y, como todo hecho social, tienen un componente de género. Entre ellos se cuentan: el descenso en el número de matrimonios, el aumento de la edad tanto de mujeres como de hombres al contraer las nupcias y el aumento de los divorcios.

La tasa de nupcialidad ${ }^{23}$ en el país viene en franca caída en las últimas décadas. En 1973 fue de 8,3; en 1991 fue de 6,8 y solo de 3,3 en 2009 (INE, 2009). La postergación del matrimonio es otro dato de marcada tendencia en los últimos años. La edad media al contraer el primer matrimonio ha aumentado notoriamente en los últimos 7 años, sobre todo en el caso de las mujeres: en 2002 las mujeres se casaban por primera vez a los 24,6 años, mientras que los hombres lo hacían a los 27,7 años. En 2009 la edad promedio en que ellas se casaban por primera vez aumentó en 4,4 años, llegando a un promedio de 29 años, mientras que en el caso de los hombres el aumento fue de 2,6 años: ahora contraen nupcias a los 30,3 años en promedio (INE, 2009). Los divorcios, por su parte, también han aumentado. En este punto se debe tener en cuenta, no obstante, que la entrada en vigencia de la ley que lo regula ${ }^{24}$ implicaba, necesariamente, la existencia de un aumento en el número absoluto de ellos en los primeros años en que rigiera. Según el Registro Civil (2012), en el año 2006 se realizaron 10.119 divorcios, cifra que aumentó a 47.076 en el año 2011. Es claro, de todos modos, que para que exista fecundidad no es condición el matrimonio. De hecho, según el Registro Civil en Chile en el año 2011 el

21. Tómese como ejemplo el permiso y el subsidio por un período determinado para cuidar a la hija o hijo menor de un año debido a una edad grave, que establece la Ley $N^{\circ} 19.250$, en su artículo 185. La ley establece que este permiso puede ser tomado por cualquiera de los padres trabajadores, sin embargo en el año 2009 en un 99,8\% de los casos este permiso fue tomado por la madre. Razones culturales relativas a la crianza, como una responsabilidad femenina, explican esta situación.

22. A simple vista parece no haber relación entre ellos y el descenso de la fecundidad, no obstante se requeriría realizar estudios para comprobar o refutar dicha interpretación.

23. Representa la frecuencia relativa de los matrimonios de una población dada en un intervalo de tiempo específico (generalmente un año civil). Su cálculo es: matrimonios celebrados en un período determinado/población media de ese período (INE, 2008).

24. Nueva Ley de Matrimonio Civil, No 19.947, promulgada en mayo de 2004. 
68,9\% de los nacimientos ocurrió fuera del matrimonio. El descenso de la tasa de nupcialidad, el aumento de los divorcios y de las hijas e hijos nacidos fuera del matrimonio indican necesariamente que la constitución de los hogares y la forma de hacer familia ha cambiado radicalmente en los últimos años. Lo que no se sabe, solo al realizar un análisis numérico de los datos poblacionales, es si las relaciones de género han cambiado (y cómo) al aumentar la conformación de hogares de otras características.

\section{CONCLUSIONES}

A todas luces los indicadores demográficos muestran que Chile está entrando en un proceso de segunda transición demográfica: se estima que los índices de fecundidad han caído a niveles inferiores al de reemplazo; ha disminuido notoriamente la tasa de matrimonios y la edad de la primera unión; han aumentado de forma radical los nacimientos de hijas(os) fuera del matrimonio y los divorcios, lo que incide en la composición distinta de los hogares. Aún falta, de todos modos, un elemento que contribuya a confirmar el ingreso de Chile a este período: la postergación de la fecha de llegada de la primera hija o hijo. Falta, además, estudiar si esta tendencia se mantendrá en el tiempo, consolidando el ingreso de Chile a esta nueva etapa demográfica.

Los hechos poblacionales que constituyen la segunda transición demográfica traen consecuencias en ambos sexos, pero de ninguna manera estas consecuencias afectan a ambos sexos por igual, pues la construcción de biografías personales y singulares se realiza de manera distinta en el proyecto de vida femenino y en el masculino. De hecho, en el caso de las mujeres el impacto puede ser aun mayor, pues tanto la disminución del número de hijas(os) como el aumento de la esperanza de vida cambiará en mayor medida la forma de estructurar sus vidas.

Para lograr comprender la segunda transición demográfica y sus alcances, se debe, por tanto, considerar siempre el enfoque de género. Es necesario incentivar tanto el estudio sobre los cambios estructurales y valóricos que están incidiendo en la transformación poblacional como el tinte de género que estos toman. De la misma manera, se requieren investigaciones que aborden cómo las relaciones de género se presentan en una sociedad, pues su expresión puede incidir en la forma que adoptan tanto los cambios sociales y culturales como los cambios poblacionales.

La utilización del enfoque de género en los asuntos poblacionales es útil también para realizar un análisis más global en cuanto a la forma de estructurar las sociedades en relación a la categoría género, pues es altamente probable que los cambios poblacionales afecten las relaciones de género que ocurren en un tiempo histórico determinado. El hecho de vivir más años, y/o de tener menos hijos e hijas, produce no solo cambios en las condiciones materiales de cada persona, sino también cambios en las significaciones y proyecciones de vida de cada una, lo que seguramente puede hacer cambiar las formas de relacionarse con el otro género. De forma recíproca, es probable que los cambios en las relaciones de género estén afectando o produciendo los cambios poblacionales. Vale entonces preguntarse: ¿los cambios de género anteceden a los cambios poblacionales o 
los cambios poblaciones provocan cambios en las relaciones de género? ¿Se produciría, debido a los cambios poblacionales, un cambio en el sistema de género imperante en Chile?

¿Permitirán los cambios poblacionales una reducción de las desigualdades de género? Las respuestas a todas estas preguntas, y a tantas otras que puedan surgir, deberán venir de la mano de un mayor desarrollo de los estudios poblacionales con enfoque de género, y, a su vez, de estudios de género que incorporen el componente poblacional.

\section{REFERENCIAS BIBLIOGRÁFICAS}

Ariza, M., De Oliveira, O., Contribuciones de la perspectiva de género a la sociología de la población en Latinoamérica, Versión preliminar para la discusión, Trabajo preparado para el panel "Repensando la Sociología Latinoamericana", XXII International Congress, Latin American Sociological Association (LASA), Miami, 2000.

Arriagada, I., "Transformaciones sociales y demográficas de las familias latinoamericanas", en Papeles de población, $\mathrm{N}^{\circ}$ 40, Toluca, México, 2004.

Beck-Gernsheim, E., La reinvención de la familia. En busca de nuevas formas de convivencia, Barcelona: Paidós, 2003.

Bernhardt, E., "Is the Second Demographic Transition a Useful Concept for Demography?", en Vienna Yearbook of Population Research Volume 2004, Vienna Institute of Demography, Austria, Viena, 2004, págs. 25-28.

Billari, F.C., Liefbroer, A. C., "Is the Second Demographic Transition a useful concept for demography? Introduction to a debate", en Vienna Yearbook of Population Research Volume 2004, Vienna Institute of Demography, Austria, Viena, 2004, págs. 1-3.

CEPAL, Observatorio demográfico No 5. Fecundidad, Santiago de Chile: CEPAL, 2008.

—- Observatorio demográfico N7. Proyección de Población, Santiago de Chile: CEPAL, 2009.

- Observatorio demográfico Nº. Mortalidad, Santiago de Chile: CEPAL, 2010.

Coleman, D., "Why we don't have to believe without doubting in the "Second Demographic Transition"-some agnostic comments", en Vienna Yearbook of Population Research Volume 2004, Vienna Institute of Demography, Austria, Viena, 2004, págs. 11-24.

Connell, R., Masculinities, Cambridge, UK: Polity Press, 1995.

Departamento de Extranjería y Migración, Bases de datos de visas e inmigración 2000-2010. Tiempo [Base de datos], Santiago de Chile, 2011.

El Mostrador, "Piñera celebra que 30 mil madres se han beneficiado con el posnatal de seis meses", 29 de febrero de 2012, véase online en http://www.elmostrador.cl/noticias/pais/2012/02/29/ pinera-celebra-que-30-mil-madres-se-han-beneficiado-con-el-posnatal-de-seis-meses/, [Consulta: 29 de febrero de 2012].

García, B., Rojas, O., "Las uniones conyugales en América Latina: Transformaciones en un marco de desigualdad social y de género", en XXIV Conferencia Internacional de la Unión Internacional para el Estudio Científico de la Población (UIECP) (XXIV, 2001, Salvador de Bahía, Brasil), 2001, págs. 65-99.

Heikel, M. V., "Género y población: un desafío más para la equidad", en Notas de población, № 62, Santiago de Chile, Centro Latinoamericano de Demografía (CELADE), 1996.

Haupt, A., Kane, T. T., Manual sobre la Población, Novena impresión, Washington, D.C, EE.UU, Population Reference Bureau, 2001. 
INE, CEPAL, Chile: Proyecciones y Estimaciones de Población. Total País. Período de Información: 1950-2050, Santiago de Chile, INE, Sin fecha.

INE, Estadísticas sociales de los pueblos indígenas en Chile. Censo 2002, Santiago de Chile, INE, 2002.

— Fecundidad en Chile. Situación reciente, Santiago de Chile, INE, 2006.

_- Encuesta Experimental de Uso del Tiempo [Base de datos], Santiago de Chile, 2007.

— Anuario de Estadísticas Vitales, Santiago de Chile, INE, 2009.

— Evolución de la mortalidad en Chile según causas de muerte y edad, 1990-2007, Santiago de Chile, INE, 2010.

— Boletín informativo, Estadísticas Vitales 2008, Santiago de Chile, INE, 2011.

Larrañaga, O., "Comportamientos Reproductivos y Natalidad: 1960-2003", en Valenzuela, J.S et al. (eds.), El eslabón perdido: Familia, modernización y bienestar en Chile, Santiago de Chile: Editorial Tauros, 2006.

Lister, R., Citizenship: Feminist Perspectives, Houndmills, Basingstoke: Macmillan, 1997.

Ministerio de Desarrollo Social, Estadísticas sobre empleo, véase online en http://www.ministeriodesarrollosocial.gob.cl/casen/Estadisticas/empleo.html, [Consulta: 22 de febrero de 2012].

Mason, K. O., Gender and Demographic Change, what do we know?, Liège, Bélgica: International Union for the Scientific Study of Population, 1995.

Medina, M., Fonseca, M. do C., Reflexiones sobre los paradigmas que explican la fecundidad, Barcelona, España, Programa ALFA-POP del Centre d'Estudis Demogràfics, Universidad Autónoma de Barcelona, 2003.

Paredes, M., Viejos problemas para nuevas cuestiones: género, procesos de individualización y segunda transición demográfica, Propuesta de trabajo a ser presentada en III Congreso de la Asociación Latinoamericana de Población, ALAP (III. 2008. Córdoba, Argentina), págs. 1-15.

PNUD, Indicadores, véase online en http://hdrstats.undp.org/es/indicadores/default.html, [Consulta: 22 de febrero de 2012].

Registro Civil e Identificación, véase online en http://www.registrocivil.cl/, [Consulta: 22 de febrero de 2012].

Stefoni, C., "Migración, género y servicio doméstico. Mujeres peruanas en Chile", en Valenzuela, M. E., Mora, C. (eds.), Trabajo doméstico: un largo camino hacia el trabajo decente, Santiago de Chile: Oficina Internacional del Trabajo, OIT, 2006. 